\title{
Decoding of Kinetic and Kinematic Information from Electrocorticograms in Sensorimotor Cortex: A Review
}

Duk Shin $^{1 *}$, Yasuhiko Nakanishi ${ }^{1}$, Chao Chen ${ }^{2}$, Hiroyuki Kambara ${ }^{1}$, Natsue Yoshimura ${ }^{1}$ and Yasuharu Koike ${ }^{1,2}$

${ }^{1}$ Precision and Intelligence Laboratory, Tokyo Institute of Technology, Yokohama, Japan

${ }^{2}$ Department of Information Processing, Tokyo Institute of Technology, Yokohama, Japan

\begin{abstract}
Brain-machine interface techniques have been applied in a number of studies to control neuromotor prostheses and for neuro-rehabilitation in the hopes of providing a means to restore lost motor function. Electrocorticography has seen recent use in this regard because it offers a higher spatiotemporal resolution than non-invasive electroencephalography and is less invasive than intracortical microelectrodes. Despite lots of successful studies; none of study has dealt with the importance of both kinematic and kinetic information for the purpose of realizing an ECoG-based neuroprosthesis.

Here, we review the decoding kinetic and kinematic information from electrocorticograms. First, we introduce our preprocessing method for decoding of muscle activities, hand trajectories, and joint angles with our previous works. Second, we review and discuss about three questions: which locations are most effective area for decoding, how different numbers of effective electrocorticography signals affect decoding performance, and which frequency band is most effective? We foresee the proposed method contributing to future advancements in neuro-prosthesis and neurorehabilitation technology.
\end{abstract}

Keywords: Brain-machine interfaces; Electrocorticography; Decoding; Muscle activity; Hand trajectory; Joint angle

\section{Introduction}

Brain-machine interfaces (BMI) are useful technologies to provide assistance to disabled individuals, allowing them interaction with their environments. A number of prominent brain-machine interface studies have arisen over the past two decades. These BMI systems translate brain signals into commands for controlling devices such as cursors [1], spelling devices [2], and neural prosthetics [3-9]. This new communication has not only the potential to help to disabled persons but also provide insight into the motor system of the brain [10-14].

Several sensors have been developed to measure brain signals. These are mainly categorized into two types, invasive sensor i.e. intracortical microelectrodes and non-invasive sensors such as electroencephalography (EEG) and magneto encephalography. Lots of invasive BMI studies have successfully demonstrated prosthetic

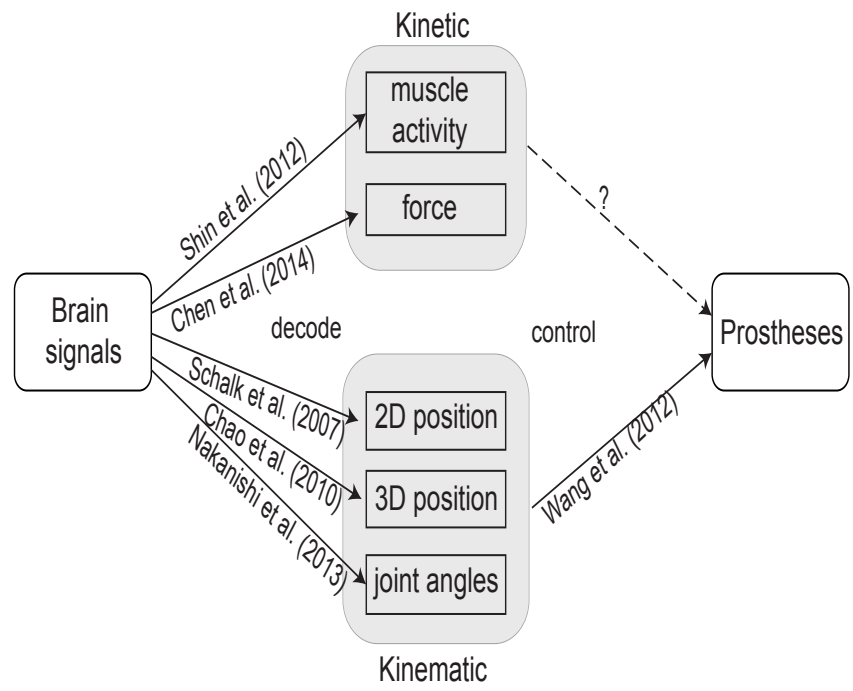

Figure 1: Schematic outline of the neuroprosthesis and the achieved ECoG based studies. devices [6-9]. However, they have the risk such as brain injury. Since EEG are non-invasive and have high temporal resolution, previous works have developed such as online cursor control [15], direction of hand movements [16,17], a spelling device [18], and neuro feedback for rehabilitation $[19,20]$. Although a large number of these non-invasive works succeeded in classification of movement intention, prediction of time-varying trajectories is difficult due to insufficient spatial resolution and low signal-to-noise ratio in such methods.

Electrocorticography (ECoG) is an alternative approach to less invasive BMIs [21-29]. ECoG is a technique that measures electrical activity in the cerebral cortex by means of electrodes placed directly on the surface of the brain. Compared to EEG, ECoG has higher spatio-temporal resolution with better signal-to-noise ratio than scalp EEG [30,31]. ECoG has also shown potential as a stable longterm recording method [27]. Several studies using ECoG have already succeeded in the classification of movement direction [22,23], grasp type [28], and prediction of hand trajectory [24,26,27], and decoding of hand trajectories [25,27,32], arm trajectories [33] and finger movement $[34,35]$. Predictions of muscle activities from ECoG signals during reaching and grasping movements in monkeys have also been successful [36]. Despite these successes, however, there still remains considerable work for the realization of ECoG-based neuroprosthesis. Since the human neuromuscular system naturally modulates mechanical stiffness and viscosity to achieve proper interaction with the environment, we have not only decoded kinematic information such as trajectory but also kinetic information such as torque, stiffness

*Corresponding author: Duk Shin, Precision and Intelligence Laboratory, Tokyo Institute of Technology, J3-10, 4259 Nagatsuda, Midori-ku, Yokohama 226-8503, Japan, Tel: +81 45924 5054; Fax: +81 45924 5085; E-mail: shinduk@cns.pi.titech.ac.jp

Received February 28, 2014; Accepted March 25, 2014; Published April 30, 2014

Citation: Shin D, NakanishiY, Chen C, Kambara H, Yoshimura N, etal. (2014)Decoding of Kinetic and Kinematic Information from Electrocorticograms in Sensorimotor Cortex. Int J Neurorehabilitation 1: 102. doi:10.4172/2376-0281.1000102

Copyright: ( $) 2014$ Shin D, et al. This is an open-access article distributed under the terms of the Creative Commons Attribution License, which permits unrestricted use, distribution, and reproduction in any medium, provided the original author and source are credited. 
and viscosity of joints. Decoding the kinematic and kinetic information from the neural activity is necessary to implement a human-like BMI system. The schematic outline of this concept and achieved studies are shown in Figure 1.

This paper introduces the preprocessing algorithm to decode the kinetic and kinematic information from ECoG signals in time series. Using this novel method, we could predict muscle activities (kinetic) and joint angles (kinematic) of shoulder and elbow joints. We also discuss three questions: which locations are most effective area for decoding, how different numbers of effective electrocorticography signals affect decoding performance, and which frequency band is most effective?

\section{A}

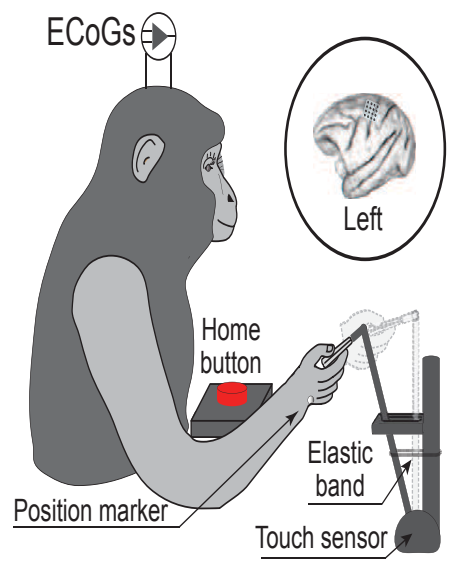

B

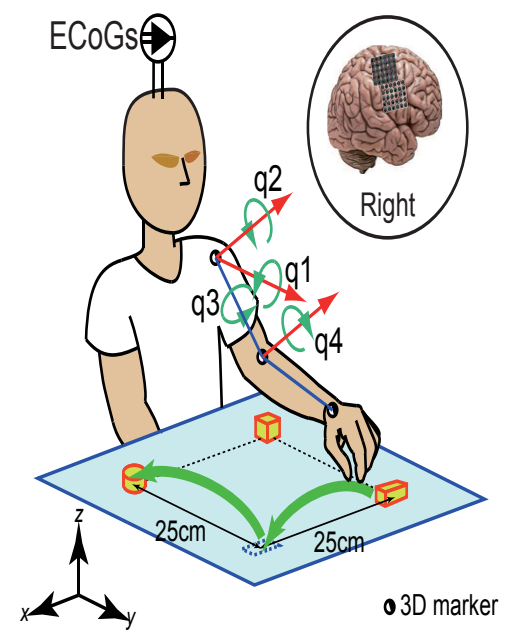

Figure 2: Behavioral task and ECoG electrode locations.

A) Monkeys performed sequential right arm and hand movements, which consisted of reaching to a knob, grasping the knob with a lateral grip, pulling the knob closer, releasing the knob, and returning the hand to the home position, in a 3-D workspace [36]. During the task, ECoG and EMG signals were recorded simultaneously.

B) Patient 1 replaced three blocks one by one and clockwise (green arrows) at the corners of a $25 \mathrm{~cm} \times 25 \mathrm{~cm}$ square [32]. ECoG signals were obtained with planar-surface platinum grid electrodes placed on the right sensorimotor cortex. Half-closed circles represent3D markers for the motion capture system. The angles q1, q2, q3, and q4 are defined as an abduction/adduction angle, a flexion/extension angle, an external/internal rotation at the left shoulder joint, and a flexion/extension angle at the left elbow joint, respectively. When he lowered his arm toward the $-z$ direction and turned his palm to the $y$ direction with the elbow extended, q1, q2, andq3 were all zero, and q4 was $\pi$ radians.

\section{Materials and Methods}

\section{Ethics statement}

We got the monkey ECoG data from the National Institutes of Natural Sciences and the human ECoG data from Osaka University Hospital in Japan. The local ethics committee of the National Institutes of Natural Sciences (Approval No.: 11A157) and Osaka University Hospital (Approval No.08061) approved each experiment. The monkeys' welfare and steps taken to ameliorate suffering were in accordance with the recommendations of the Weather all report, "The use of non-human primates in research." Human experiment conducted in accordance with the Declaration of Helsinki. ECoG electrodes were embedded not for our experiments but for patients' medical treatments. The ECoG arrays were implanted in the intracranium for two weeks to determine the optimum site for effective pain reduction (patients 1 and 2) or epileptic foci localization (patient 3). All patients or their guardians gave written informed consent for the use of their data in the academic study.

Experiment 1: Monkey data: Two Japanese macaques (Monkey A: male, at $8.9 \mathrm{~kg}$; Monkey B: female, at $4.7 \mathrm{~kg}$ ) were trained to perform reaching and grasping tasks with the right hand as shown in Figure 2A. The monkeys performed these tasks repeatedly and continuously for over $700 \mathrm{~s}$. Monkey A performed a total of 134 trials, and monkey B performed 248 trials. We chronically implanted a platinum ECoG array (Unique Medical Corporation, Tokyo, Japan) over the left M1, which contained 15 (monkey A: $5 \times 3$ grid) and 16 (monkey B: $4 \times 4$ grid) channel electrodes.

We recorded ECoG signals with $4 \mathrm{kHz}$ sampling using an acquisition processor system (Plexon MAP System; Plexon, Inc., Dallas, US) and EMG activities of the right forelimb muscles implanted pairs of multistranded stainless steel wires (Cooner Wire, Chatsworth, CA, USA). The 3D-positions of various points of the right arm were recorded using reflective markers tracked with an optical motion capture system (Eagle Digital System; Motion Analysis Corporation, Santa Rosa, CA). The neural data were down-sampled to 500 samples per second, and the motion data were up-sampled to 500 samples per second to match the neural data. The previous work showed the detail experimental information [36].

Experiment 2: Human data: All patients were seated upright on a chair at a table and were asked to perform the tasks using their left hands as shown in Figure 1B. They asked to replace three blocks to vacant corners of the square around a $25 \mathrm{~cm} \times 25 \mathrm{~cm}$, one by one in a clockwise fashion (patient 1), random choose (patient 2), and an arbitrary positioning (patient 3 ). Patients 1 and 2 were implanted with two $5 \times 6$ electrode arrays, and patient 3 was implanted with a $3 \times 5$ array. ECoG signals were recorded inside an electromagnetically shielded room with a 128-channel digital EEG system (EEG 2000; Nihon Koden Corporation, Tokyo, Japan) set at a sampling rate of $1000 \mathrm{~Hz}$. 3D arm motions were recorded at a sampling rate of $100 \mathrm{~Hz}$ with an optical motion capture system (Eagle Digital System; Motion Analysis Corporation, Santa Rosa, CA). Nakanishi et al. [32] showed the experimental setup in detail.

Decoding method: ECoG signals were pre-processed with our previously proposed method $[32,33,36]$. Firstly, the signal data were rereferenced with a common average reference $(\mathrm{CAR})$ and divided into seven or nine frequency bands $(d: \sim 4 \mathrm{~Hz}, q: 4 \sim 8 \mathrm{~Hz}, a: 8 \sim 14 \mathrm{~Hz}$, $b 1: 14 \sim 20 \mathrm{~Hz}, b 2: 20 \sim 30 \mathrm{~Hz}, \gamma 1: 30 \sim 50 \mathrm{~Hz}$, and $\gamma 2: 50 \sim 90 \mathrm{~Hz}$, $\gamma 3$ : $90 \sim 120 \mathrm{~Hz}$, and $\gamma 4: 120 \sim 150 \mathrm{~Hz}$ ) using fourth-order band pass Butterworth filters. Secondly, these band-passed signals were digitally 
Citation: Shin D, Nakanishi Y, Chen C, Kambara H, Yoshimura N, et al. (2014) Decoding of Kinetic and Kinematic Information from Electrocorticograms in Sensorimotor Cortex. Int J Neurorehabilitation 1: 102. doi:10.4172/2376-0281.1000102

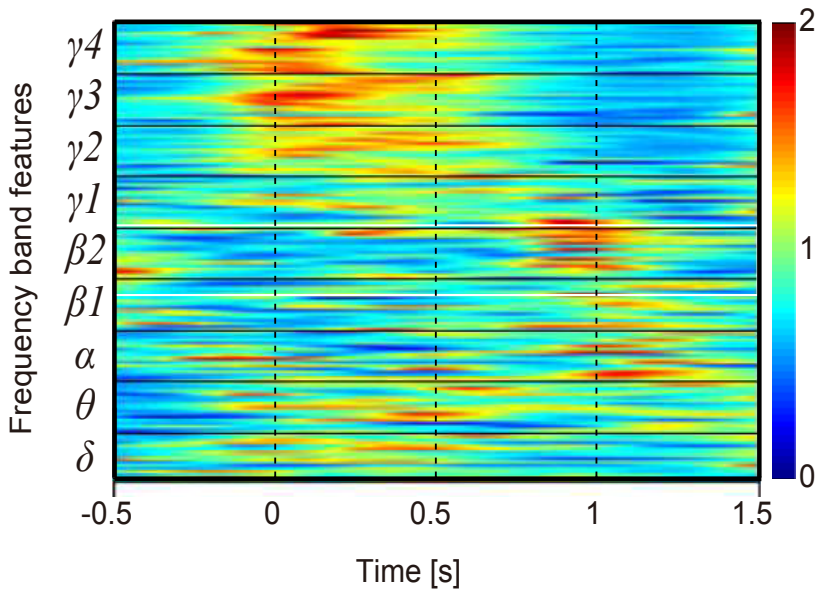

Figure 3: Example of ECoG spectral feature during a movement task. Frequency band feature data were sorted into channels and frequency bands [33].

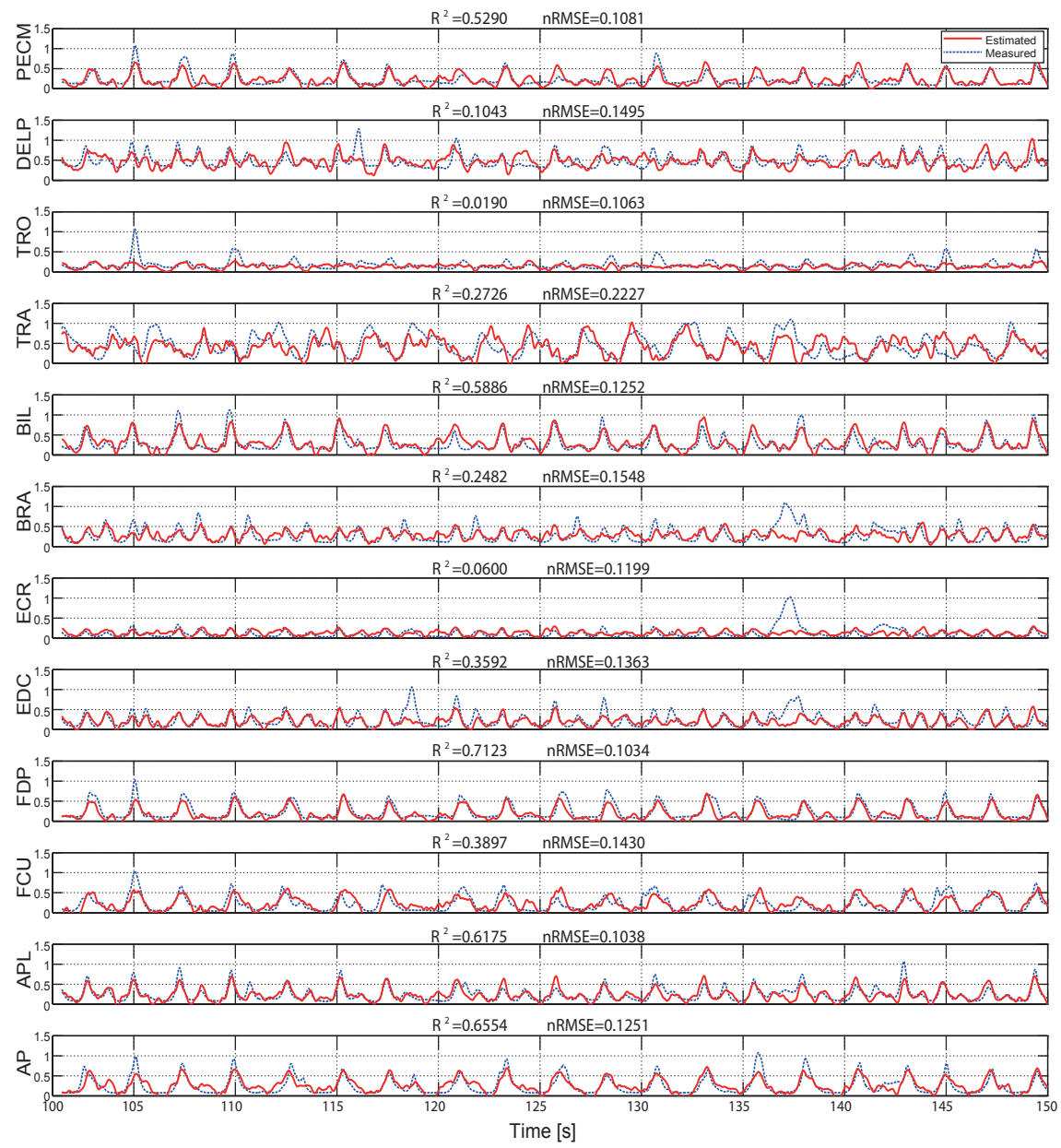

Figure 4: Example of muscle activity prediction in a continuous time series.

Dotted blue lines are actual muscle activities from EMG signals and solid red lines are decoded muscle activities from ECoG signals over a $50 \mathrm{~s}$ time interval

[36]. Both lines were normalized to the ranges of actual muscle activities. The normalized root mean square error (nRMSE) and coefficient of determine ( $\mathrm{R}^{2}$ ) are also shown.

rectified and smoothed with a Gaussian filter (width: $0.1 \mathrm{~s}, \sigma: 0.04 \mathrm{~s}$ ), which changed high oscillations into low frequency features. Thirdly, the signals were down sampled to $100 \mathrm{~Hz}$, i.e., the sampling rate of the motion capture recordings. Finally, the obtained signals $x_{i}(t)(\mathrm{i}=1,2, \ldots$ $, \mathrm{n} \times 7$ or $\mathrm{n} \times 9)$ at time $\mathrm{t}$ were normalized to the standard $\mathrm{z}$-score $\mathrm{z}_{\mathrm{i}}(\mathrm{t})$ as follows. 


$$
z_{i}(t)=\frac{x_{i}(t)-\mu_{i}}{\sigma_{i}} \quad(\mathrm{i}=1,2, \ldots, \mathrm{n} \times 7 \text { or } \mathrm{n} \times 9)
$$

where $m, s_{i}$ and $\mathrm{n}$ denote the mean value of $x_{i}(t)$, the standard deviation of $x_{i}(t)$, and the number of ECoG channels, respectively. These z-scores calculated from ECoG signals were utilized as training data to construct a decoder. Figure 3 shows an example trial including frequency band features of the ECoG signals, rectified raw EMG signals, grip force, and logical signals. We used the sparse linear regression (SLiR) or the Partial least squares regression (PLS) algorithm to determine the weight for prediction.

\section{Results}

\section{Decoding of muscle activities from ECoG signals}

The neuromuscular system naturally modulates mechanical stiffness and viscosity of arm to achieve proper interaction force to the environments. Stiffness, viscosity and force of joints change

A

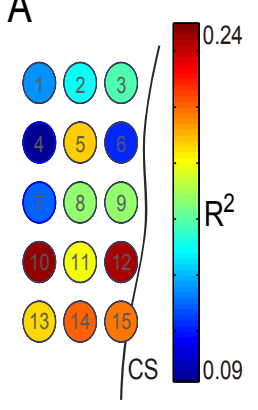

B

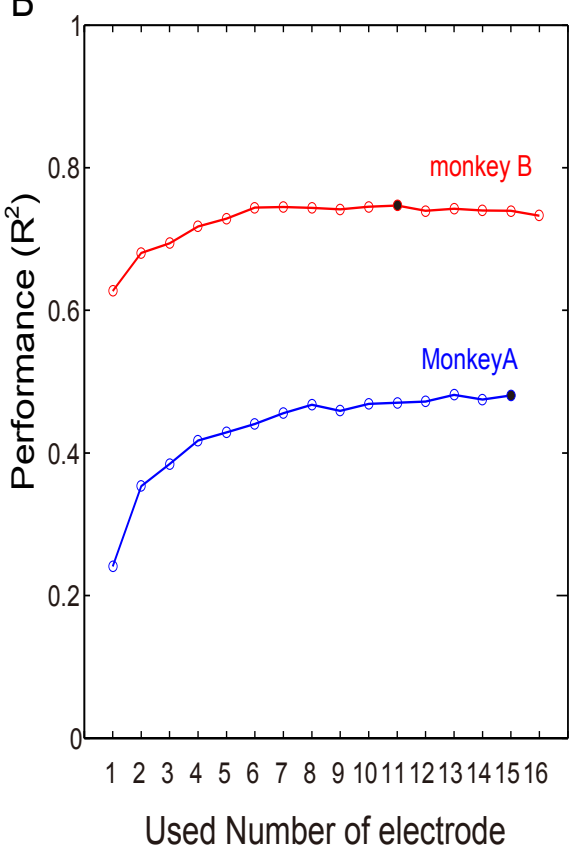

Figure 5: Decoding results for each individual electrode and performance vs. used number of electrodes.

A) Prediction performance for each electrode. The color map in each electrode represents performance of that electrode.

$B$ ) The blue line represents the decoding performance for monkey $A$. The red line represents the decoding performance for monkey $B$. The filled circles denote the highest performances.

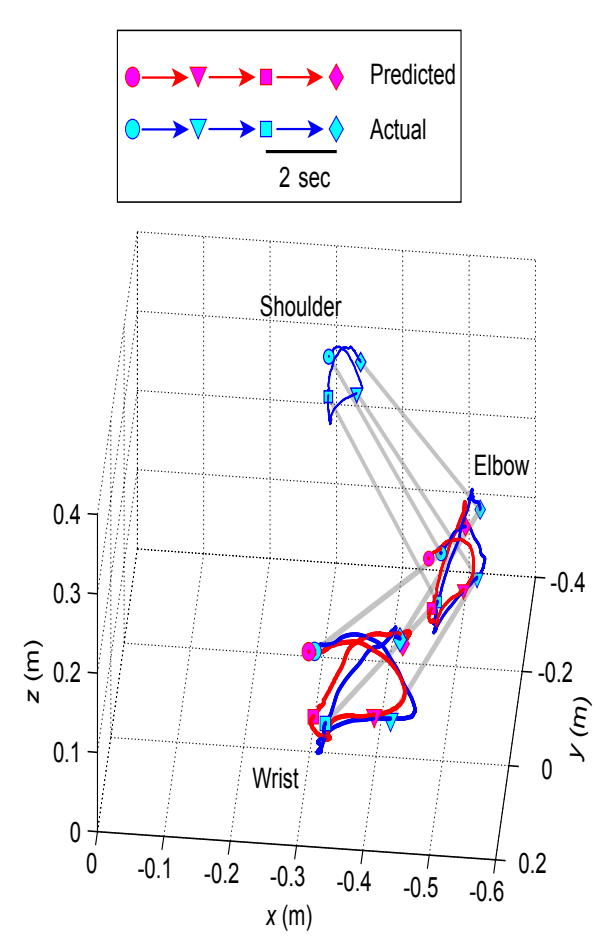

Figure 6: Examples of the predicted and actual 3D trajectories [32].

Markers (circles, triangles, squares, and diamonds) represent $2 \mathrm{~s}$ time intervals. Circles and diamonds indicate the earliest and the latest positions, respectively. The red trajectories were computed using predicted data q1 q4 and patient 1's actual arm length. The timings (positions of the markers) and trajectory curves of the predicted data were similar to those of the actual data.

with muscle activation. Therefore, decoding muscle activities are key components for realizing neuro-prosthesis capable of the interaction with environments. We verified that ECoG signals are effective for predicting muscle activities in time varying series when performing sequential movements [36]. We used sparse linear regression to find the best fit between frequency bands of ECoG and electromyographic activity. We applied the prediction model to continuous data from an additional session by monkey $B$. One example of continuous prediction is shown in Figure 4, where the prediction was stable even for repetitive trials over 50s. In the results of the 5-cross validation, Mean and standard deviation (STD) of the coefficient of determination $\left(\mathrm{R}^{2}\right)$ and nRMSE for each muscle ranged from $0.02 \pm 0.006$ to $0.63 \pm 0.003\left(R^{2}\right)$ and 0.13 \pm 0.005 to $0.18 \pm 0.01$ (nRMSE). These results could demonstrate the feasibility of predicting muscle activity from ECoG signals in an online fashion. Recently, we succeeded in decoding grasp force profile during reaching and grasping tasks [37].

\section{Decoding of hand trajectory from ECoG signals}

We also succeed in decoding 3 dimensional hand positioning from ECoG signals using the proposed preprocessing algorithm and PLS regression [33]. To determine the most effective areas for prediction, we calculated performance values $\left(\mathrm{R}^{2}\right)$ using only individual electrode. Performance details of two electrode selection methods are shown in Figure 5. For both monkeys, performance was improved quickly as the number of electrodes used increased from 1 to 6 . The performance curves fluctuated only slightly when using 9 electrodes and above. The best $\mathrm{R}^{2}$ values were achieved using 15 and 11 electrodes for monkeys $A$ and $B$, respectively. Higher performance electrodes are concentrated at the lateral areas and near areas of central sulcus (CS). Our results 
indicated that $3 \mathrm{D}$ hand trajectories can be predicted using nine or ten ECoG signals and that ECoG electrodes with higher performance were concentrated at the lateral areas and areas close to CS.

\section{Decoding of joint angles from ECoG signals}

We also predicted 3D angle trajectories in time series from ECoG signals in humans using theproposed preprocessing method and a sparse linear regression [32]. Figure 6 is an example of the comparison between predicted (red lines) and actual 3D trajectories (blue lines) for six seconds in the 10th trial of session 2 by patient 1 .

\section{Discussion}

\section{Most effective location for decoding}

Carmena et al. [10] reported that neuron activity recorded from $\mathrm{Ml}$ showed greater efficacy than that from dorsal premotor cortex, supplementary motor cortex, posterior parietal cortex, and primary somatosensory cortex. In our previous work [33], it is clearly shown that the electrodes in primary motor area are most contributing to decode among the premotor area, primary sensory area, and primary motor areaas shown in Figure 4A-4D. Within primary motor area, however, we could not find experimental evidences to explain the most effective site for force prediction according to anatomical knowledge. Our results just found that ECoG signals from the lateral areas and near areas of CS showed greater efficacy in prediction [33,36]. It might be needed the micro-sized ECoG electrode to find the most effective location within primary motor area.

\section{Most effective number of electrodes}

For both monkeys, performance improved quickly as the number of electrodes used increased from 1 to 9 as shown in figure $4 \mathrm{E}$. The performance curves fluctuated only slightly when using 10 electrodes and above. Best decoding performance was achieved using a relatively small number of electrodes, 13 and 10 electrodes in the performancebased selection for monkey A and monkey B, respectively. These trends are similar to the results of a previous neuron activity-based study [38], which selected different numbers of high sensitivity neurons in decoding kinematic variables. We note that decoding performance is not simply related to the number of electrode but may more closely depend on the higher density electrodes within the effective areas. Nevertheless, a small number of electrodes would allow for lower power consumption, extending the usage time for wireless ECoG-based BMIs $[39,40]$.

\section{Most effective frequency band for decoding}

Most EEG-based BMI studies have used one or two sensorimotor rhythms such as $\mu(8 \sim 12 \mathrm{~Hz})$ or $\beta(14 \sim 30 \mathrm{~Hz})$ oscillations because the $\gamma(>30 \mathrm{~Hz})$ rhythm is often inconspicuous and neglected with a low pass filter. In ECoG-based BMIs, however, the $\gamma$ rhythm has been widely used. We identified the useful ECoG frequency bands to decode kinetic and kinematic information. Analysis of the weight values for the frequency bands showed that contributions by the $\delta, \gamma$, and $\beta$ bands were significantly larger than those of the other bands $[33,36]$. This result corresponds to previous studies as well [27,41-45]. Especially, the $\gamma$ band was most effective than any other bands because $\gamma$ band activity of ECoG signals reflect the unit activity in layers V/VI in primary motor area [46].

\section{Conclusion}

This study introduced the novel attempt to decode muscle activities, hand trajectories, and joint angles from a small number of ECoG signals. This approach offers important insight regarding the presence of kinetic and kinematic information in ECoG signals to predict timevarying their information, whereas previous ECoG-based studies have tried to classify direction or intention of movement. The primary advantage of the proposed method is that it can predict muscle activities and joint angle during sequential movement tasks. If we can predict muscle activities, joint torque and stiffness can also be predicted using previously proposed methods $[47,48]$. This creates remarkable benefits, which would contribute to the realization of ECoG-based prosthetics. We foresee this method contributing to future advancements in neuroprosthesis and neuro-rehabilitation technology.

\section{Acknowledgment}

A part of this study was the result of "Brain Machine Interface Development" carried out under the Strategic Research Program for Brain Sciences by the Ministry of Education, Culture, Sports, Science and Technology of Japan. We thank Watanabe M, Isa T, Nishimura Y (NIPS) and Yanagisawa T, Hirata M (Osaka University Medical School) for providing ECoG data.

\section{References}

1. Wolpaw JR, McFarland DJ, Neat GW, Forneris CA (1991) An EEG-based braincomputer interface for cursor control. Electroencephalogr Clin Neurophysiol 78 252-259.

2. Birbaumer N, Ghanayim N, Hinterberger T, Iversen I, Kotchoubey B, et al (1999) A spelling device for the paralysed. Nature 398: 297-298.

3. Chapin JK, Moxon KA, Markowitz RS, Nicolelis MA (1999) Real-time contro of a robot arm using simultaneously recorded neurons in the motor cortex. Nat Neurosci 2: 664-670.

4. Wessberg J, Stambaugh CR, Kralik JD, Beck PD, Laubach M, et al. (2000) Real-time prediction of hand trajectory by ensembles of cortical neurons in primates. Nature 408: 361-365.

5. Taylor DM, Tillery SI, Schwartz AB (2002) Direct cortical control of 3D neuroprosthetic devices. Science 296: 1829-1832.

6. Hochberg LR, Serruya MD, Friehs GM, Mukand JA, Saleh M, et al. (2006) Neuronal ensemble control of prosthetic devices by a human with tetraplegia. Nature 442: 164-171.

7. Velliste M, Perel S, Spalding MC, Whitford AS, Schwartz AB (2008) Cortical control of a prosthetic arm for self-feeding. Nature 453: 1098-1101.

8. Hochberg LR, Bacher D, Jarosiewicz B, Masse NY, Simeral JD, et al. (2012) Reach and grasp by people with tetraplegia using a neurally controlled robotic arm. Nature 485: 372-375.

9. Wang W, Collinger JL, Degenhart AD, Tyler-Kabara EC, Schwartz AB, et al. (2013) An electrocorticographic brain interface in an individual with tetraplegia. PLoS One 8: e55344.

10. Carmena JM, Lebedev MA, Crist RE, O'Doherty JE, Santucci DM, et al. (2003) Learning to control a brain-machine interface for reaching and grasping by primates. PLoS Biol 1: E42.

11. Wolpaw JR, McFarland DJ (2004) Control of a two-dimensional movement signal by a noninvasive brain-computer interface in humans. Proc Natl Acad Sci U S A 101: 17849-17854.

12. Lebedev MA, Carmena JM, O'Doherty JE, Zacksenhouse M, Henriquez CS, et al. (2005) Cortical ensemble adaptation to represent velocity of an artificial actuator controlled by a brain-machine interface. J Neurosci 25: 4681-4693.

13. Fagg AH, Hatsopoulos NG, de Lafuente V, Moxon KA, Nemati S, et al. (2007) Biomimetic brain machine interfaces for the control of movement. J Neurosci 27: 11842-11846.

14. Hauschild M, Mulliken GH, Fineman I, Loeb GE, Andersen RA (2012) Cognitive signals for brain-machine interfaces in posterior parietal cortex include continuous 3D trajectory commands. Proc Natl Acad Sci U S A 109: 1707517080.

15. Wolpaw JR, McFarland DJ, Neat GW, Forneris CA (1991) An EEG-based braincomputer interface for cursor control. Electroencephalogr Clin Neurophysiol 78: 252-259. 
Citation: Shin D, Nakanishi Y, Chen C, Kambara H, Yoshimura N, et al. (2014) Decoding of Kinetic and Kinematic Information from Electrocorticograms in Sensorimotor Cortex. Int J Neurorehabilitation 1: 102. doi:10.4172/2376-0281.1000102

16. Blankertz B, Dornhege G, Krauledat M, Müller KR, Kunzmann V, et al. (2006) The Berlin Brain-Computer Interface: EEG-based communication without subject training. IEEE Trans Neural Syst Rehabil Eng 14: 147-152.

17. Waldert S, Preissl H, Demandt E, Braun C, Birbaumer N, et al. (2008) Hand movement direction decoded from MEG and EEG. J Neurosci 28: 1000-1008.

18. Birbaumer N, Ghanayim N, Hinterberger T, Iversen I, Kotchoubey B, et al. (1999) A spelling device for the paralysed. Nature 398: 297-298.

19. Birbaumer N, Cohen LG (2007) Brain-computer interfaces: communication and restoration of movement in paralysis. J Physiol 579: 621-636.

20. Daly JJ, Wolpaw JR (2008) Brain-computer interfaces in neurological rehabilitation. Lancet Neurol 7: 1032-1043.

21. Leuthardt EC, Schalk G, Wolpaw JR, Ojemann JG, Moran DW (2004) A braincomputer interface using electrocorticographic signals in humans. J Neural Eng 1: 63-71.

22. Pistohl T, Ball T, Schulze-Bonhage A, Aertsen A, Mehring C (2008) Prediction of arm movement trajectories from ECoG-recordings in humans. J Neurosci Methods 167: 105-114.

23. Levine SP, Huggins JE, BeMent SL, Kushwaha RK, Schuh LA, et al. (2000) A direct brain interface based on event-related potentials. IEEE Trans Rehabil Eng 8: 180-185.

24. Mehring C, Nawrot MP, de Oliveira SC, Vaadia E, Schulze-Bonhage A, et al. (2004) Comparing information about arm movement direction in single channels of local and epicortical field potentials from monkey and human motor cortex. Journal of Physiology-Paris 98: 498-506.

25. Schalk G, Kubánek J, Miller KJ, Anderson NR, Leuthardt EC, et al. (2007) Decoding two-dimensional movement trajectories using electrocorticographic signals in humans. J Neural Eng 4: 264-275.

26. Sanchez JC, Gunduz A, Carney PR, Principe JC (2008) Extraction and localization of mesoscopic motor control signals for human ECoG neuroprosthetics. J Neurosci Methods 167: 63-81.

27. Chao ZC, Nagasaka Y, Fujii N (2010) Long-term asynchronous decoding of arm motion using electrocorticographic signals in monkeys. Front Neuroeng 3: 3.

28. Pistohl T, Schulze-Bonhage A, Aertsen A, Mehring C, Ball T (2012) Decoding natural grasp types from human ECoG. Neuroimage 59: 248-260.

29. Parameshwaran D, Crone NE, Thiagarajan TC (2012) Coherence potentials encode simple human sensorimotor behavior. PLoS One 7: e30514.

30. Ball T, Kern M, Mutschler I, Aertsen A, Schulze-Bonhage A (2009) Signal quality of simultaneously recorded invasive and non-invasive EEG. Neuroimage 46: 708-716.

31. Slutzky MW, Jordan LR, Krieg T, Chen M, Mogul DJ, et al. (2010) Optimal spacing of surface electrode arrays for brain-machine interface applications. J Neural Eng 7: 26004

32. Nakanishi Y, Yanagisawa T, Shin D, Fukuma R, Chen C, et al. (2013) Prediction of three-dimensional arm trajectories based on ECoG signals recorded from human sensorimotor cortex. PLoS One 8: e72085.

33. Chen C, Shin D, Watanabe H, Nakanishi Y, Kambara H, et al. (2013) Prediction of hand trajectory from electrocorticography signals in primary motor cortex. PLoS One 8: e83534.
34. Kubánek J, Miller KJ, Ojemann JG, Wolpaw JR, Schalk G (2009) Decoding flexion of individual fingers using electrocorticographic signals in humans. $J$ Neural Eng 6: 066001.

35. AcharyaS, Fifer MS, Benz HL, Crone NE, Thakor NV (2010) Electrocorticographic amplitude predicts finger positions during slow grasping motions of the hand. $\mathrm{J}$ Neural Eng 7: 046002.

36. Shin D, Watanabe H, Kambara H, Nambu A, Isa T, et al. (2012) Prediction of muscle activities from electrocorticograms in primary motor cortex of primates. PLoS One 7: e47992.

37. Chen C, Shin D, Watanabe H, Nakanishi Y, Kambara H, et al. (2014) Decoding grasp force profile from electrocorticographysignals in non-human primate sensorimotor cortex. Neuroscience Research. 83: 1-7.

38. Sanchez JC, Carmena JM, Lebedev MA, Nicolelis MAL, Harris JG, et al. (2004) Ascertaining the importance of neurons to develop better brain-machine interfaces. IEEE Transactions on Biomedical Engineering 51: 943-953.

39. Bjorninen T, Muller R, Ledochowitsch P, Sydanheimo L, Ukkonen L, et al. (2012) Design of wireless links to implanted brain-machine interface microelectronic systems. IEEE Antennas and Wireless Propagation Letters 11: 1663-1666.

40. Hirata M, Matsushita K, Suzuki T, Yoshida T, Sato F, Morris S, et al. (2011) A fully-implantable wireless system for human brain-machine interfaces using brain surface electrodes: W-HERBS. IEICE Trans Commun. E94-B: 2448-2453.

41. Flint RD, Ethier C, Oby ER, Miller LE, Slutzky MW (2012) Local field potentials allow accurate decoding of muscle activity. J Neurophysiol 108: 18-24.

42. Stark E, Abeles M (2007) Predicting movement from multiunit activity. J Neurosci 27: 8387-8394.

43. Flint RD, Lindberg EW, Jordan LR, Miller LE, Slutzky MW (2012) Accurate decoding of reaching movements from field potentials in the absence of spikes. J Neural Eng 9: 046006.

44. Zhuang J, Truccolo W, Vargas-Irwin C, Donoghue JP (2010) Decoding 3-D reach and grasp kinematics from high-frequency local field potentials in primate primary motor cortex. IEEE Trans Biomed Eng 57: 1774-1784.

45. Bansal AK, Truccolo W, Vargas-Irwin CE, Donoghue JP (2012) Decoding 3D reach and grasp from hybrid signals in motor and premotor cortices: spikes, multiunit activity, and local field potentials. J Neurophysiol 107: 1337-1355.

46. Yazdan-Shahmorad A, Kipke DR, Lehmkuhle MJ (2013) High gamma power in ECoG reflects cortical electrical stimulation effects on unit activity in layers V/ VI. J Neural Eng 10: 066002.

47. Shin D, Kim J, Koike Y (2009) A myokinetic arm model for estimating joint torque and stiffness from EMG signals during maintained posture. J Neurophysiol 101 387-401.

48. Kawase T, Kambara H, Koike Y (2011) A Power Assist Device Based on Joint Equilibrium Point Estimation from EMG Signals. Journal of Robotics and Mechatronics 24: 205-218. 